\title{
Periods of a Moduli Space of Bundles on Curves
}

\section{Citation}

Mumford, David B., and P. Newstead. 1968. Periods of a moduli space of bundles on curves. American Journal of Mathematics 90(4): 1200-1208.

\section{Published Version}

doi:10.2307/2373296

\section{Permanent link}

http://nrs.harvard.edu/urn-3:HUL.InstRepos:3597250

\section{Terms of Use}

This article was downloaded from Harvard University's DASH repository, and is made available under the terms and conditions applicable to Other Posted Material, as set forth at http:// nrs.harvard.edu/urn-3:HUL.InstRepos:dash.current.terms-of-use\#LAA

\section{Share Your Story}

The Harvard community has made this article openly available.

Please share how this access benefits you. Submit a story.

Accessibility 


\section{PERIODS OF A MODULI SPACE OF BUNDLES ON CURVES.}

By D. Mumford and P. Newstead.

We will work over the complex numbers in this paper. For all curves $C$, and for all integers $(n, d)$, the problem arises of determining the structure of the "space" of all vector bundles $E$, with rank $n$ and degree $\left(=\operatorname{deg} c_{1}(E)\right) d$. The problem has been considerably clarified recently by the introduction of the concept of stable and semi-stable bundles: [4], [6], [10]. It has been proven, in particular, that for each $n$ and each line bundle $L$ on $C$ such that $n$ and $\operatorname{deg} L$ are relatively prime, then the set:

$$
S_{n, L}(C)=\left\{\begin{array}{l}
\text { set of all stable vector bundles } E \text { on } C \text { of } \\
\operatorname{rank} n \text { such that } \Lambda^{n} E \cong L
\end{array}\right.
$$

has a natural structure of a non-singular projective variety of dimension $\left(n^{2}-1\right) \cdot(g-1)$, where $g=$ genus $(C)$. It is important to note that the map

$$
E \mapsto E \otimes M
$$

for a line bundle $M$ induces an isomorphism

$$
S_{n, L}(C) \stackrel{\approx}{\longrightarrow} S_{n, L} \otimes \mathbb{M}^{n}(C)
$$

hence the variety $S_{n, L}(C)$ depends essentially only on the residue class of $\operatorname{deg} L \bmod n$.

We wish to look at the case $g \geqq 2, n=2, \operatorname{deg} L$ odd. In this case, we may assume for simplicity that a base point $x_{0} \in C$ has been chosen that $L$ is taken to be the line bundle whose sections form the sheaf $O_{-C}\left(x_{0}\right)$. We abbreviate $S_{2, L}(C)$ now to $S_{2}{ }^{-}(C)$. The topology of these varieties has been described in [\%] and when the genus of $C$ is 2 , their complete structure is described in [8]. $S_{2}^{-}(C)$ has dimension $3 g-3$ and is known to be birationally equivalent to $\boldsymbol{P}_{3 g-3}$. In particular, it is simply connected and the invariants $h^{0, p}=h^{p, 0}$ are all $0,([9])$. In [ [ ] $]$, it is also proven that $B_{2}=1$, $B_{3}=2 g$. Now for non-singular projective varieties $X$ with $h^{0,3}=h^{3,0}=0$, a very interesting invariant is Weil's "intermediate jacobian" attached to $H^{3}(X)$. This is an abelian variety, which we shall denote $J^{2}(X)$, which is by definition:

Received June 12, 1967. 


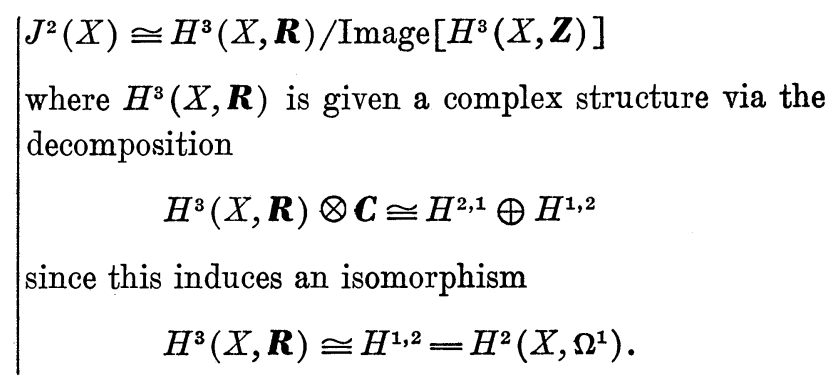

cf. [11], [1], [3]. Weil also showed that a polarization on $X$ induces a polarization on $J^{2}(X)$ in a canonical way.

If $\operatorname{Alb}(C)$ denotes the albanese, or jacobian, variety of $C$, then our main result is :

Theorem. $J^{2}\left[S_{2}^{-}(C)\right] \cong \operatorname{Alb}(C)$.

Note that $S_{2}^{-}(C)$ has a unique polarization since $B_{2}=1$, hence $J^{2}\left(S_{2}^{-}-(C)\right)$ has a canonical polarization, just as $\operatorname{Alb}(C)$ does. It is easy to check that our isomorphism is compatible with these canonical polarizations, hence by Torelli's theorem, we conclude:

Corollary. If $S_{2}^{-}\left(C_{1}\right) \cong S_{2}^{-}\left(C_{2}\right)$, then $C_{1} \cong C_{2}$.

Before beginning the proof, we must recall Weil's map relating $J^{2}(X)$ to codimension 2 cycles on $X$ :

let $Y$ be an non-singular parameter space,

let $W$ be an algebraic cycle on $X \times Y$ of codimension 2 .

Then we get

$w \in H^{4}(X \times Y, \boldsymbol{Z})$, the fundamental class of $W$

esp. $w_{3,1} \in\left(H^{3}(X, \boldsymbol{Z}) /\right.$ torsion $) \otimes H^{1}(Y, \boldsymbol{Z})$, the $(3,1)$-component

of $w$.

Then $w_{3,1}$ defines a map

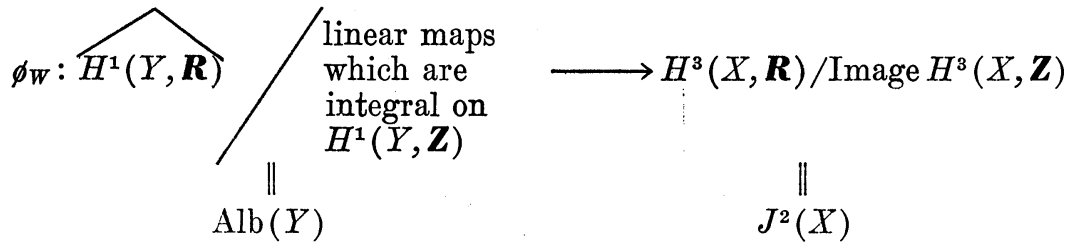


which is easily seen to be complex-analytic using the fact that $w$ is of type $(2,2)$ in the Hodge decomposition of $H$. Note the obvious fact:

LEMMA 1. $\phi_{W}$ is an isomorphism if and only if $w_{3,1}$ is "unimodular," (i.e., written out as a matrix in terms of bases of $H^{3}(X, \mathbf{Z}) /$ torsion, $H^{1}(Y, \mathbf{Z})$, it is a square matrix with det $= \pm 1$ ).

1. In the sequel, we abbreviate $S_{2}-(C)$ by $S$. The first step in our proof is to construct a universal vector bundle $E$ on $S \times C$, i. e., one whose restriction to $\{t\} \times C$, for any $t \in S$, is exactly the vector bundle $E_{t}$ on $C$ corresponding to the point $t \in S$. This is a problem in descent theory. In fact, $S$ can be described as a quotient $R / P G L(v)$, where $R$ is a non-singular quasi-projective variety, and $P G L(v)$ acts freely on $R$; and where there is a vector bundle $F$ on $R \times C$ whose restriction to $\{t\} \times C$, any $t \in R$, is the vector bundle on $C$ corresponding to the image of $t$ in $S:$ cf. [10], p. 321. However, the difficulty is that the action of $P G L(v)$ on $R$ does not, a priori, lift to an action on $F$. Instead, $G L(v)$ acts on $F$ satisfying

1) $\boldsymbol{G}_{m}=$ center $(G L(v))$ acts on $F$ by homotheties

2) if $\pi: G L(v) \rightarrow P G L(v)$ is the canonical map, and $T_{g}$ represents the action of an element $g$, then the diagram

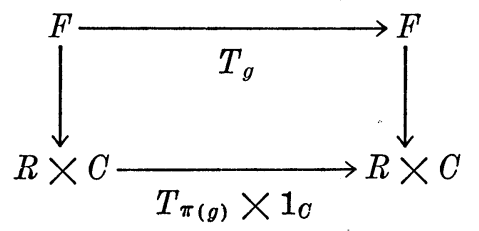

commutes.

The way out of this type of impasse is to find a "functorial" way of associating to every vector bundle $E$ on $C$ (of the type being considered) a 1 dimensional vector space $\lambda(E)$ such that multiplication by $\alpha$ in $E$ induces multiplication by $\alpha$ in $\lambda(E)$. By functorial we mean that the procedure extends to families of such vector bundles: if $E$ is a vector bundle on $T \times C$ (for any algebraic scheme $T$ ) whose restriction to $\{t\} \times C$ is of the type under consideration, then we should get a line bundle $\lambda(E)$ on $T$. Moreover, for any diagram of vector bundles

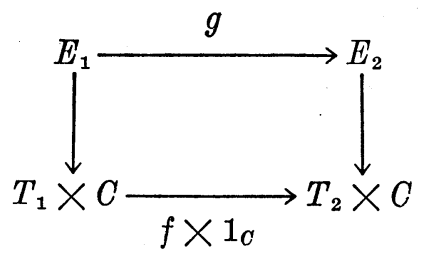


making $E_{1}$ into a fibre product of $E_{2}$ and $T_{1} \times C$ over $T_{2} \times C$, we should be given a definite isomorphism of $\lambda\left(E_{1}\right)$ with $f^{*}\left(\lambda\left(E_{2}\right)\right)$. For example, if $T_{1}=T_{2}=\operatorname{Spec}(\boldsymbol{C}), E_{1}=E_{2}=E$, and $g$ is multiplication by a scalar $\alpha \neq 0$, we are then given an induced automorphism of $\lambda(E)$ : we want this automorphism to be multiplication by $\alpha$ too (it might turn out to be multiplication by $\alpha^{n}$ instead). All this data is subject to an obvious co-cycle condition: compare [5], p. 64. If we can find such data, we get as a consequence a line bundle $\lambda(F)$ on $R$, plus an action of $G L(v)$ on $\lambda(F)$ in which the center acts by homotheties. If we then define

$$
F^{\prime}=F \otimes p_{1}^{*}\left(\lambda(F)^{-1}\right),
$$

we get a new vector bundle on $R \times C$ with the same restrictions to the fibres $\{t\} \times C$ as before; but where in the natural action of $G L(v)$ on $F^{\prime}$, the action of the center $\boldsymbol{G}_{m}$ on $F$ and $p_{1}^{*}\left(\lambda(F)^{-1}\right)$ cancel each other out, i. e., $P G L(v)$ acts on $F$. Then $F / P G L(v)$ is the sought-for universal vector bundle on $S \times C$.

Here's how to construct $\lambda$. We limit ourselves to the case $T=\operatorname{Spec}(\boldsymbol{C})$, $E$ a vector bundle on $C$, since the generalization of $\lambda$ to an arbitrary base will be clear. Recall $E$ has rank 2, degree 1, and is stable:

a) $H^{1}\left(E \otimes\left(\Omega_{C^{1}}\right)^{k}\right)=(0)$, if $k \geqq 1$.

Proof. This group is dual to $H^{0}\left(\hat{E} \otimes\left(\Omega_{O}^{1}\right)^{1-k}\right)$ and if this were nonzero, we would get a non-zero homomorphism

$$
\left(\Omega_{C^{1}}\right)^{k-1} \rightarrow \hat{E}
$$

hence a sub-line-bundle $G \subset \hat{E}$ of degree $\geqq 2(k-1)(g-1) \geqq 0$. This contradicts the stability of $E$.

b) If $V_{k}(E)=H^{0}\left(E \otimes\left(\Omega_{O^{1}}\right)^{k}\right)$, then

$$
\operatorname{Dim} V_{k}(E)=(2 g-2)(2 k-1)+1 \text {. }
$$

Proof. Riemann-Roch.

c) Set $\lambda(E)=\left[\Lambda^{2 g-1} V_{1}(E)\right]^{\otimes(3 g-1)} \otimes\left[\Lambda^{6 g-5} V_{2}(E)\right]^{\otimes(-g)}$. Then multiplication by $\alpha$ in $E$ induces the endomorphism, multiplication by $\alpha$, in each $V_{k}(E)$, hence it induces multiplcation by $\alpha$ to the power 


$$
(3 g-1)(2 g-1)+(6 g-5)(-g)
$$

in $\lambda(E)$. This number happens to be 1 !

We now know that $E$ exists. Next consider the chern classes of $E$. We have

$$
\begin{aligned}
c_{2}(E) \in H^{4}(S \times C, \boldsymbol{Z}) & \cong\left(H^{2}(S, \boldsymbol{Z}) \otimes H^{2}(C, \mathbf{Z})\right) \\
c_{1}(E) \in H^{2}(S \times C, \boldsymbol{Z}) & \cong H^{2}(C, \mathbf{Z}) \otimes H^{2}(S, \mathbf{Z}) \\
& \otimes\left(H^{3}(S, \boldsymbol{Z}) \otimes H^{1}(C, \mathbf{Z})\right) \\
& \otimes H^{4}(S, \mathbf{Z}) .
\end{aligned}
$$

Note that any bundle $E \otimes p_{1} * M, M$ a line bundle on $S$, would have the same universal property that $E$ does, so $c_{1}(E)$ is not very interesting. However, let

$$
\alpha=\left(c_{2}(E)\right)_{3,1}=\left[\text { component of } c_{2}(E) \text { in } H^{3}(S, \boldsymbol{Z}) \otimes H^{1}(C, \boldsymbol{Z})\right] \text {. }
$$

A simple computation of chern classes shows that $\alpha$ is independent of this modification of $E$. According to $[7], H^{3}(S, \boldsymbol{Z})$ and $H^{1}(C, \boldsymbol{Z})$ have the same rank. In fact:

\section{Proposition 1. $\alpha$ is unimodular.}

This will be proven in $\S 2$. Assuming this, it follows from Lemma 1 that if $W=$ the algebraic 2nd chern class of $E$, then Weil's map $\phi_{W}: \operatorname{Alb}(C) \rightarrow J^{2}(S)$ is an isomorphism, as required. Although it is not essential, it will be convenient in $\S 2$ to know that $H^{3}(S, \boldsymbol{Z})$ is torsion-free. In fact, the torsion subgroup of $H^{3}(X, \mathbf{Z})$-for any non-singular complete variety $X$ over $\boldsymbol{C}$-is a birational invariant of $X$ known as the "topological Brauer group" (cf. [12], Cor. (\%.3) and equation (8.9), p. 59). And $S$ is birationally equivalent to $\boldsymbol{P}_{3 g-3}$ which has no $H^{3}$ at all!

2. We start by recalling the results of [6]. In fact, let $S_{0}$ be the subset of $S U(2)^{2 g}$ consisting of points $\left(A_{1}, \cdots, A_{2 g}\right)$ such that

$$
\prod_{i=1}^{g} A_{2 i-1} A_{2 i} A_{2 i-1}^{-1} A_{2 i}^{-1}=-I .
$$

Then $S_{0}$ is an orientable submanifold of $S U(2)^{2 g}$ and there is a natural map

$$
p: S_{0} \rightarrow S,
$$

which is a principal fibration with group $P U(2)$. The map $p$ may be determined as follows. Let $\tilde{C}$ be the simply-connected covering of $C$ which is 
ramified over $x_{0}$ with ramification index 2 . The group $\pi$ of this covering is generated by elements $a_{1}, \cdots, a_{2 g}$ subject to the single relation

$$
\left[\prod_{i=1}^{g} a_{2 i-1} a_{2 i} a_{2 i-1}{ }^{-1} a_{2 i^{-1}}\right]^{2}=e .
$$

Thus a point of $S_{0}$ may be regarded as a representation of $\pi$, and this representation defines a stable bundle $E$ over $C$ of $\operatorname{rank} 2$, with $\Lambda^{2} E \cong L$, and hence a point of $S$. So we get a map $p: S_{0} \rightarrow S$. Notice that the $a_{i}$ determine elements of $\pi_{1}(C)$ and hence of $H_{1}(C ; \mathbf{Z})$, and that these elements form a basis for $H_{1}(C ; \mathbf{Z})$; let $\left\{\alpha_{i}\right\}$ be the dual basis of $H^{1}(C ; \mathbf{Z})$.

LEMma 2. $p^{*}: H^{3}(S ; \mathbf{Z}) \rightarrow H^{3}\left(S_{0} ; \mathbf{Z}\right)$ is an isomorphism.

Proof. Since $H^{1}(P U(2) ; \boldsymbol{Z})=0$ and $H^{1}(S ; \mathbf{Z})=0$ ( $S$ is simply-connected), the spectral sequence of the fibration $p$ gives rise to an exact sequence

$$
H^{0}\left(S ; H^{2}(P U(2) ; \mathbf{Z})\right) \rightarrow H^{3}(S ; \mathbf{Z}) \stackrel{p^{*}}{\longrightarrow} H^{3}\left(S_{0} ; \mathbf{Z}\right) \rightarrow H^{0}\left(S ; H^{3}(P U(2) ; \mathbf{Z})\right) .
$$

Now the first group in this sequence is $\boldsymbol{Z}_{2}$ and the last is $\boldsymbol{Z}$. Moreover $H^{3}(S ; \boldsymbol{Z})$ is torsion-free (see $\S 1$ ) and has the same rank as $H^{3}\left(S_{0} ; \mathbf{Z}\right)$ by the results of $[7]$. The lemma now follows.

Lemma 3. The homomorphism $H^{3}\left(S U(2)^{2 g}, \mathbf{Z}\right) \rightarrow H^{3}\left(S_{0} ; \boldsymbol{Z}\right)$ induced by the inclusion of $S_{0}$ in $S U(2)^{2 g}$ is an isomorphism.

Proof. Lemma 3 of [y] shows that the homomorphism

$$
H_{3}\left(S_{0} ; \mathbf{Z}\right) \rightarrow H_{3}\left(S U(2)^{2 g}, \mathbf{Z}\right)
$$

is surjective, except possibly for some 2-primary torsion. However, in this simple case, the same argument can be used to prove that the homomorphism is really surjective. It follows at once that $H^{3}\left(S U(2)^{2 g} ; \boldsymbol{Z}\right)$ is contained in $H^{3}\left(S_{0} ; \boldsymbol{Z}\right)$ as a direct summand. The lemma now follows from the fact that the ranks of these two groups are equal (see [\%]) and that $H^{3}\left(S_{0} ; \mathbf{Z}\right.$ ) is torsion-free by Lemma 2 .

Now let $p_{i}: S_{0} \rightarrow S U(2)$ denote the projection on the $i$-th factor and let

$$
\beta_{i}=p_{i}^{*}\left[\text { generator of } H^{3}(S U(2) ; \mathbf{Z})\right] \text {. }
$$

Then by Lemma 3 the $\beta_{i}$ form a basis for $H^{\mathbf{3}}\left(S_{0} ; \boldsymbol{Z}\right)$. In view of Lemma 2 , it is now sufficient to prove:

Proposition 2. $\quad c_{2}\left[\left(p \times 1_{C}\right) * E\right]_{3,1}=\sum_{i=1}^{2 g} \beta_{i} \otimes \alpha_{i}$. 
Now choose embedding $s_{i}: S^{1} \rightarrow C-x_{0}$ which represent the generators $a_{i}$ of $\pi$. Then

$$
\begin{aligned}
s_{i}^{*}\left(\alpha_{j}\right) & =0 & & i \neq j \\
& =\text { generator of } H^{1}\left(S^{1} ; \boldsymbol{Z}\right) & & i=j .
\end{aligned}
$$

Hence Proposition 2 will follow at once from

Proposition 3.

$$
c_{2}\left[\left(1_{S_{0}} \times s_{i}\right)^{*}\left(p \times 1_{\sigma}\right)^{*} E\right]_{3,1}=\beta_{i} \otimes\left[\text { generator of } H^{1}\left(S_{.}^{1} ; \mathbf{Z}\right)\right] .
$$

We now need to recall a few more details from [6]. Let $E_{\rho}$ be the bundle over $C$ corresponding to the representation $\rho \in S_{0}$. Then ([6] Remark 6.2) we can write down coordinate transformations for $E_{\rho}$ as follows. Choose a finite open covering $\left\{U_{i}\right\}(i=0,1, \cdots, m)$ of $C$ such that every non-empty intersection of the sets $U_{i}$ is contractible. Assume $x_{0} \in U_{0}, x_{0} \notin U_{i}$ for $i \neq 0$. Assume moreover that there exist discs $D_{i}$ in $\tilde{C}$ such that $U_{0}$ is the quotient of $D_{0}$ by $\boldsymbol{Z}_{2}$ and that for $i \neq 0, D_{i}$ maps homeomorphically onto $U_{i}$. For every $i, j, k$, where $k=i$ or $j$, let $W_{i j, k}$ be a connected component of $v^{-1}\left(U_{i} \cap U_{j}\right) \cap D_{k}$ (where $v: \tilde{C} \rightarrow C$ is the covering map). If $U_{i} \cap U_{j}=\emptyset$, $i \neq j, W_{i j, k}$ maps homeomorphically onto $U_{i} \cap U_{j}$; let $\gamma_{i j}$ be the element of $\pi$ such that $\gamma_{i j} W_{i j, j}=W_{j i, i}$. Then a set of coordinate transformations $g_{i j}$ for $E_{\rho}$ is given by

$$
\begin{aligned}
g_{i j} & =\rho\left(\gamma_{i j}\right) \text { on } U_{i} \cap U_{j}, i \neq 0, j \neq 0 \\
& =f_{i} \cdot \rho\left(\gamma_{0 i}\right) \text { on } U_{0} \cap U_{i}, i \neq 0,
\end{aligned}
$$

where $f_{i}$ is an analytic scalar function on $U_{0} \cap U_{i}$ which is independent of $\rho$. Note that the coordinate transformations depend differentially on $\rho$, so that the same $g_{i j}$ (now regarded as functions on $S_{0} \times U_{i} \cap S_{0} \times U_{j}$ ) define a differentiable bundle $E^{\prime}$ over $S_{0} \times C$ which is a differentiable family of analytic bundles over $C$.

Now $E^{\prime}\left|\{\rho\} \times C \cong E_{\rho} \cong\left(p \times 1_{o}\right) * E\right|\{\rho\} \times C$ for all $\rho \in S_{0}$. Since $E$ is stable, it follows that

$$
\operatorname{dim} H^{0}\left(C ; \underset{\text { Anal. }}{\operatorname{Hom}}\left(E^{\prime},\left(p \times 1_{C}\right)^{*} E\right) \mid\{\rho\} \times C\right)=1
$$

for all $\rho$. So by Proposition 2.7 of [2],

$$
\bigcup_{\rho \in S_{0}} H^{0}\left(C ; \underset{\text { Anal. }}{\operatorname{Hom}}\left(E^{\prime},\left(p \times 1_{O}\right)^{*} E\right) \mid\{\rho\} \times C\right)
$$


has a natural structure of differentiable line bundle over $S_{0}$. Let $L$ be the induced line bundle over $S_{0} \times C$. There is then an obvious isomorphism

So

$$
E^{\prime} \otimes L \cong\left(p \times 1_{c}\right) * E
$$

$$
c_{2}\left[\left(p \times 1_{\sigma}\right) * E\right]_{3,1}=c_{2}\left(E^{\prime}\right)_{3,1} \text {. }
$$

Using the above explicit description of the bundle $E^{\prime}$, we see that for any continuous map $s: S^{1} \rightarrow C-x_{0},\left(1_{S_{0}} \times s\right) * E^{\prime}$ can be described as follows: take a trivial bundle of rank 2 over $S_{0} \times[0,1]$ and glue its ends together by means of the map

defined by

$$
S_{0} \rightarrow S U(2)
$$

$$
\rho \mapsto \rho(a) \text {, where } a \in \pi \text { corresponds to } s \text {. }
$$

Apply this when $s=s_{i}, a=a_{i}$, and $\rho\left(a_{i}\right)=p_{i}(\rho)$; so Proposition 3 will follow at once from

Lemma 4. Let $W$ be a space and let $F$ be the bundle over $W \times S^{1}$ obtained by glueing together the two ends of the trivial bundle of rank 2 over $W \times[0,1]$ by means of the map $f: W \rightarrow S U(2)$. Then

$c_{2}(F)=f^{*}\left[\right.$ generator of $\left.H^{3}(S U(2) ; \boldsymbol{Z})\right] \otimes\left[\right.$ generator of $\left.H^{1}\left(S^{1} ; \boldsymbol{Z}\right)\right]$.

Proof. $F$ is the bundle induced by $f$ from the bundle obtained by taking $W=S U(2), f=1_{S U(2)}$ in the construction. Hence it is sufficient to prove the lemma for this special case. But then it follows from the fact that $H^{*}(B S U(2))$ is generated by $c_{2}$.

This completes the proof of Proposition 3 and hence of our theorem.

\section{REFERENCES.}

[1] P. Griffiths, "Periods of integrals on algebraic manifolds," I, II, III, forthcoming. [2] K. Kodaira and D. C. Spencer, "On deformations of complex analytic structures," Annals of Mathematics, vol. 67 (1958), p. 328.

[3] D. Lieberman, "Higher Picard varieties," forthcoming. 
[4] D. Mumford, "Projective invariants of projective structures," International Mathematical Congress, Stockholm, 1962, p. 526.

[5] - " "Picard groups of moduli problems," Proc. Conf. Arith. Alg. Geom., Harper and Row, 1966.

[6] M. S. Narasimhan and C. S. Seshadri, "Stable and unitary vector bundles on a compact Riemann surface," Annals of Mathematics, vol. 82 (1965), p. 540.

[7] P. E. Newstead, “Topological properties of some spaces of stable bundles," Topology, vol. 6 (1967), p. 241.

[8] — " Stable bundles of rank 2 and odd degree over a curve of genus 2," forthcoming.

[9] J. P. Serre, "On the fundamental group of a unirational variety," Journal of the London Mathematical Society, vol. 34 (1959), p. 481.

[10] C. S. Seshadri, "Space of unitary vector bundles on a compact Riemann surface," Annals of Mathematics, vol. 84 (1967), p. 303.

[11] A. Weil, "Sur la variété de Picard," American Journal of Mathematics, vol. 74 (1952), p. 865.

[12] A. Grothendieck, Le groupe de Brauer III, mimeographed notes from Institut Hautes Etudes Sciences. 